\title{
Multidimensional MRI for characterization of subtle axonal injury accelerated using an adaptive nonlocal multispectral filter
}

Dan Benjamini ${ }^{1,2,3, *}$, Mustapha Bouhrara ${ }^{4}$, Michal E. Komlosh ${ }^{1,2,3}$, Diego lacono $^{3,5-9}$, Daniel P. Perl ${ }^{5,7}$, David L. Brody ${ }^{2,10}$, and Peter J. Basser ${ }^{1,2}$

${ }^{1}$ Section on Quantitative Imaging and Tissue Sciences, National Institute of Child Health and Human Development, National Institutes of Health, Bethesda, MD, USA

${ }^{2}$ Center for Neuroscience and Regenerative Medicine, Uniformed Services

University of the Health Sciences, Bethesda, MD, USA

${ }^{3}$ The Henry M. Jackson Foundation for the Advancement of Military Medicine (HJF), Bethesda, MD, USA

${ }^{4}$ Magnetic Resonance Physics of Aging and Dementia Unit, National Institute of Aging, National Institutes of Health, Baltimore, MD, USA

${ }^{5}$ Brain Tissue Repository \& Neuropathology Program, Uniformed Services

University (USU), Bethesda, MD, USA

${ }^{6}$ Department of Neurology, F. Edward Hébert School of Medicine, Uniformed Services University, Bethesda, MD, USA

${ }^{7}$ Department of Pathology, F. Edward Hébert School of Medicine, Uniformed Services University, Bethesda, MD, USA

${ }^{8}$ Department of Anatomy, Physiology and Genetics, F. Edward Hébert School of Medicine, Uniformed Services University, Bethesda, MD, USA

${ }^{9}$ Neurodegeneration Disorders Clinic, National Institute of Neurological Disorders and Stroke, National Institutes of Health, Bethesda, MD, USA

${ }^{10}$ Laboratory of Functional and Molecular Imaging, National Institute of Neurological Disorders and Stroke, National Institutes of Health, Bethesda, MD, USA

Correspondence*:

Dan Benjamini, National Institute of Child Health and Human Development, National Institutes of Health, Bethesda, MD, 20814, USA

dan.benjamini@nih.gov

\section{ABSTRACT}

Multidimensional MRI is an emerging approach that simultaneously encodes water relaxation $\left(T_{1}\right.$ and $T_{2}$ ) and mobility (diffusion) and replaces voxel-averaged values with subvoxel distributions of those MR properties. While conventional (i.e., voxel-averaged) MRI methods cannot adequately quantify the microscopic heterogeneity of biological tissue, using subvoxel information allows to selectively map a specific $T_{1}-T_{2}$-diffusion spectral range that corresponds to a group of tissue elements. The major obstacle to the adoption of rich, multidimensional MRI protocols for diagnostic or monitoring purposes is the prolonged scan time. Our main goal in the present study is to evaluate the performance of a nonlocal estimation of multispectral magnitudes (NESMA) filter on reduced datasets to limit the total acquisition time required for reliable multidimensional $\mathrm{MRI}$ characterization of the brain. Here we focused and reprocessed results from a recent study that identified potential imaging biomarkers of axonal injury pathology from the joint analysis of multidimensional MRI, in particular voxelwise $T_{1}-T_{2}$ and diffusion- $T_{2}$ spectra in human Corpus 
Callosum, and histopathological data. We tested the performance of NESMA and its effect on the accuracy of the injury biomarker maps, relative to the co-registered histological reference. Noise reduction improved the accuracy of the resulting injury biomarker maps, while permitting data reduction of $35.7 \%$ and $59.6 \%$ from the full dataset for $T_{1}-T_{2}$ and diffusion- $T_{2}$ cases, respectively. As successful clinical proof-of-concept applications of multidimensional MRI are continuously being introduced, reliable and robust noise removal and consequent acquisition acceleration would advance the field towards clinically-feasible diagnostic multidimensional MRI protocols.

Keywords: multidimensional, MRI, diffusion, relaxation, traumatic brain injury, axonal injury, multispectral nonlocal filtering, NESMA

\section{INTRODUCTION}

Water molecules within biological tissues interact with their local chemical environment via nuclear relaxation processes and follow diffusion patterns trajectories that are governed by the local tissue density and geometry. Using a combination of magnetic field profiles to probe these mechanisms, magnetic resonance (MR) provides exquisite sensitivity to both the chemical composition, through relaxation parameters, and microstructure, through diffusion parameters, of biological tissues.

One fundamental obstacle for using MRI to characterize tissue heterogeneity is the averaging that occurs across the image volume elements, known as voxels (i.e., pixels with thickness). Voxel-averaged images can only provide macroscopic information with respect to the voxel size, which is typically $\sim 1-3 \mathrm{~mm}^{3}$. In a mammalian brain, an individual voxel contains multiple chemical and physical microenvironments such as axons, neurons, glia, myelin, and cerebrospinal fluid. Many biological processes-of-interest take place at a microscopic scale that only affects a small portion of any given voxel, which therefore makes them undetectable using conventional voxel-averaged MRI methods. The inability to separate normal and pathological tissue within a voxel is a major contributor to the insensitivity and ensuing non-specificity of conventional MRI methods in detecting abnormal cellular processes.

By simultaneously encoding multiple MR “dimensions", such as relaxation times $\left(T_{1}\right.$ and $\left.T_{2}\right)[1]$ and diffusion [2, 3], multidimensional distributions of those MR parameters can provide fingerprints of various chemical and physical microenvironments within the volume-of-interest, which can be traced back to specific materials and cellular components. If combined with imaging [4], multidimensional MRI has the potential to overcome the voxel-averaging limitation by accomplishes two fundamental goals: (1) it provides unique intra-voxel distributions instead of an average over the whole voxel; this allows identification of multiple components within a given voxel [5, 6, 7], while (2) the multiplicity of dimensions inherently facilitates their disentanglement; this allows higher accuracy and precision in derived quantitative values $[8,9,10,11]$.

Although traditionally multidimensional MR experiments required many repeated acquisitions and therefore have imposed serious time constraints [12], acquisition strategy [13, 14], computational [15, 6 , 3, 16], and pulse design [17, 18] technological breakthroughs have significantly reduced the data burden and positioned multidimensional MRI as a powerful emerging imaging modality for studying biological media. Despite of these advances, wide-spread clinical translation still presents challenges, in particular, due to relatively low signal-to-noise ratio (SNR) and the ensuing increased data amount requirement. To address that, we report the use of a nonlocal estimation of multispectral magnitudes (NESMA) filter [19] on multidimensional MRI data to perform noise reduction for reliable parameter determination and further data reduction. To date, NESMA has been successfully used to improve determination of myelin water 


\section{METHOD}

fraction from multi-spin-echo MR images [20], or cerebral blood flow from arterial spin labeling MR images [21].

We chose to focus and reprocess a subset of data from our recent study that showed multidimensional MRI can uncover subtle axonal injury patterns in the human brain, otherwise inaccessible using conventional quantitative MRI techniques such as diffusion tensor imaging (DTI), $T_{1}$ or $T_{2}$ maps [22]. The study investigated brain samples derived from human subjects who had sustained traumatic brain injury (TBI) and control brain donors using MRI, followed by co-registered histopathology that included amyloid precursor protein (APP) immunoreactivity to define axonal injury severity [23]. Abnormal multidimensional $T_{1}-T_{2}$, mean diffusivity- $T_{2}\left(\mathrm{MD}-T_{2}\right)$, and $\mathrm{MD}-T_{1}$ spectral signatures that were strongly correlated with injured voxels were identified and used to generate axonal injury biomarker maps [22]. Here we study the effect of applying a multispectral nonlocal filter on three representative cases (a control and two TBI cases), with the main goal of evaluating the performance of NESMA on reduced datasets to limit the total acquisition time required for reliable multidimensional MRI characterization of brain tissue. The co-registered APP histology images serve as a "ground truth" reference, thus providing a unique opportunity to quantitatively evaluate to what extent the accuracy of the injury biomarkers maps is preserved under substantial data reduction.

\subsection{Donors specimens employed in the present study}

We evaluated autopsy-derived brain specimens from two different human brain collections. Formalin-

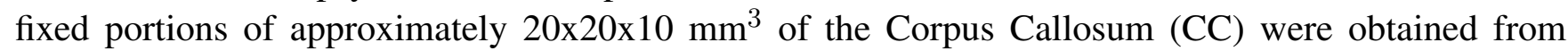
one military subject from the DoD/USU Brain Tissue Repository and Neuropathology Program (https://www.researchbraininjury.org, Bethesda, MD; Subject 1), and two civilian subjects enrolled in the Transforming Research and Clinical Knowledge in Traumatic Brain Injury study (TRACK-TBI; https://tracktbi.ucsf.edu/transforming-research-and-clinical-knowledge-tbi) (Subjects 2 and 3). For each case, a next-of-kin or legal representative provided a written consent for donation of the brain for use in research. The brain tissues used have undergone procedures for donation of the tissue, its storage, and use of available clinical information that have been approved by the USU Institutional Review Board (IRB) prior to the initiation of the study. All experiments were performed in accordance with current federal, state, DoD, and NIH guidelines and regulations for postmortem analysis.

Subject 1 was a 44 years old male with no known TBI history and postmortem APP-negative histopathology. Subject 2 was a 60 year old male that died as a result of a intraparenchymal hemorrhage following a motor vehicle accident. Subject 3 was a 49 year old male that died as a result of intraparenchymal and subarachnoid hemorrhages following a fall.

\subsection{MRI acquisition}

Prior to MRI scanning, each formalin-fixed brain specimen was transferred to a phosphate-buffered saline (PBS) filled container for 12 days to ensure that any residual fixative was removed from the tissue. The specimen was then placed in a $25 \mathrm{~mm}$ tube, and immersed in perfluoropolyether (Fomblin LC/8, Solvay Solexis, Italy), a proton free fluid void of a proton-MRI signal. Specimens were imaged using a 7 T Bruker vertical bore MRI scanner equipped with a microimaging probe and a $25 \mathrm{~mm}$ quadrupole RF coil.

Multidimensional data were acquired using a 3D echo planar imaging (EPI) sequence with a total of 56 and 302 images for $T_{1}-T_{2}$ and MD- $T_{2}$, respectively, and with $300 \mu \mathrm{m}$ isotropic spatial resolution, which resulted in respective acquisition times of 4.5 and $17.8 \mathrm{hr}$. To test the feasibility of data reduction using NESMA we derived reduced datasets by sub-sampling the full datasets. The total number of $T_{1}-T_{2}$ images 
was reduced from 56 to 36 (35.7\% decrease), while the total number of MD- $T_{2}$ images was reduced from 302 to 122 (59.6\% decrease). Further details can be found in the Supplementary Material.

The SNR was always maintained above 100 (defined as the ratio between the average unattenuated signal intensity within a tissue region of interest, and the standard deviation of the signal intensity within the background). The sample temperature was set at $16.8^{\circ} \mathrm{C}$.

\subsection{Multidimensional MRI processing}

Here we implemented a marginally-constrained, $\ell_{2}$-regularized, nonnegative least square optimization to compute the multidimensional distribution in each voxel, as previously described [8, 24]. It is a well-tested approach that had been proved robust and reliable [25, 26, 27, 2, 28, 29, 14], which in this study had resulted in two types of distributions in each voxel: $T_{1}-T_{2}$ and MD- $T_{2}$. The $2 \mathrm{D} T_{1}-T_{2}$ and MD- $T_{2}$ distributions were evaluated on $50 \times 50$ logarithmically sampled grids using a previously described algorithm [13]. The range for $T_{1}$ was $1-10,000 \mathrm{~ms}$, the range for $T_{2}$ was $1-500 \mathrm{~ms}$, and the range for MD was $0.0001-5 \mu \mathrm{m}^{2} / \mathrm{ms}$.

If one considers the multidimensional distributions as spectra, it is possible to use them to generate maps of specific spectral components by means of integration over a predefined parameter range generally associated with a spectral peak. The integral value is a number between 0 and 1 , representing a certain spectral component (SC) in a given multidimensional distribution, which can be computed in each voxel to generate an image of that specific SC [30]. Here we apply a recently proposed unsupervised algorithm to identify the injury-associated spectral information [22], and generate injury biomarker maps that closely follow APP histopathology.

\subsection{The nonlocal estimation of multispectral magnitudes (NESMA) filter}

For each sample, the multidimensional distributions were derived from the original multidimensional data as well as from data denoised using the NESMA filter to improve accuracy and precision in derived distributions.

We consider $K$ multidimensional images defined on a discrete grid describing the 3D spatial domain spanned by the image. The underlying idea of quantitative filters is to reduce noise by replacing the intensity of a given voxel by an unbiased estimate of its underlying amplitude. This requires selection of voxels that are likely to come from similar tissue. The NESMA filter restores the amplitude, $A$, of an index voxel, $i$, based on intensities of $M$ preselected voxels with similar multispectral signal patterns through:

$$
A_{k}(i)=\frac{1}{M} \sum_{j}^{M} S_{k}(j),
$$

where $S_{k}(j)$ is the measured amplitude in voxel $j$ of frame $k . M$ is the total number of similar voxels defined using the relative Manhattan distance (RMD) between voxel intensities as

$$
R M D(i, j)=100 \times \frac{\sum_{k=1}^{K}\left|S_{k}(i)-S_{k}(j)\right|}{\sum_{k=1}^{K} S_{k}(i)} .
$$

The RMD was calculated between the index voxel $i$ and all voxels belonging to a relatively large search window of size $R$, centered around the index voxel $i$, in which emission and reception $B_{1}$ fields and noise standard deviation (SD) were assumed to be approximately constant. The size of the window must be sufficiently large to ensure inclusion of an adequate number of similar voxels, and sufficiently restricted to ensure that the transmission and reception $B_{1}$ fields and noise SD are approximately constant within 


\section{RESULTS} can be found in [22]. study.

the window. In this work, we used a relatively conservative window size to avoid introducing bias in the estimated amplitudes. The size of the search window, $R$, was fixed at $11 \times 11 \times 11$ voxels. Voxels with $\mathrm{RMD}<5 \%$ were considered similar to the index voxel.

\subsection{Histopathology}

After MRI scanning, each CC tissue block was transferred for histopathological processing. Tissue blocks from each brain specimen were processed using an automated tissue processor (ASP 6025, Leica Biosystems, Nussloch, Germany). After tissue processing, each tissue block was embedded in paraffin and cut in a series of $5 \mu \mathrm{m}$-thick consecutive sections on which immunohistochemistry for anti-amyloid precursor protein (APP) was performed (DS9800, Leica Biosystems, Buffalo Grove, IL). Further details

\subsection{Quantification of axonal damage}

Images of APP stained sections were digitized using an Aperio whole slide scanning scanner system (Leica Biosystems, Richmond, IL) at $\times 20$ magnification. The following steps, all implemented using MATLAB (The Mathworks, Natick, MA), were taken to allow for a quantitative analysis of the APP images. First, the images were transformed into a common, normalized space to enable improved quantitative analysis [31]. Then, the normalized images were deconvolved to unmix the primary (APP) and secondary (hematoxylin and eosin, H\&E) stains, and background to three separate channels [32]. Once an APP-only image was obtained, a final thresholding step was taken to exclude non-specific staining and to allow for a subsequent $\%$ area calculation.

From each tissue section, based on APP staining, traumatic axonal injury (TAI) lesions were identified by an experienced neuropathologist (DI) as white matter (WM) areas with swollen axonal varicosities, axonal bulbs, or distorted axons. Accordingly, regions of interest (ROI) of normal-appearing WM and TAI lesions were manually defined. Additionally, gray matter (GM) ROIs from adjacent cingulate cortex were defined in all sections. Twelve ROIs, covering together an average $81 \mathrm{~mm}^{2}$ of tissue, were identified per tissue section. After extracting the ROIs, APP density was expressed as the percentage of total area within the ROI in the binary deconvolved APP image. In total, 36 ROIs from three subjects were included in this

\subsection{Axonal injury spectral signatures are preserved after filtering}

We first investigated the spatially-resolved subvoxel $T_{1}-T_{2}$ and MD- $T_{2}$ spectral components to assess the effect of NESMA on the derived voxelwise spectra. To do that, it is useful to summarize the 4D information, which consists of $2 \mathrm{D}$ images with $50 \times 50$ spectra in each voxel, as arrays of images with varying subvoxel $T_{1}, T_{2}$, and MD values. To make them more readable, the $50 \times 50$ spectra were sub-sampled on a 10 $\times 10$ grid. These maps are shown in Figs. 1, 2, and 3 for all three Subjects. Corresponding histological APP images (co-registered with the MRI) are shown on the left panel of Fig. 4, with red color indicating abnormal APP accumulation.

Starting with the control case (Subject 1), the spatially-resolved subvoxel $T_{1}-T_{2}$ and MD- $T_{2}$ spectral components are shown in Fig. 1. The left column shows the results from the unfiltered data $\left(T_{1}-T_{2}\right.$ and $\mathrm{MD}-\mathrm{T}_{2}$ in Figs. 1 $\mathrm{A}$ and $\mathrm{C}$, respectively), while the right column shows the results from the filtered data ( $T_{1}-T_{2}$ and $\mathrm{MD}-T_{2}$ in Figs. $1 \mathrm{~B}$ and $\mathrm{D}$, respectively). The maps revealed signal components that were spatially consistent with specific tissue types such as white matter and gray matter. 


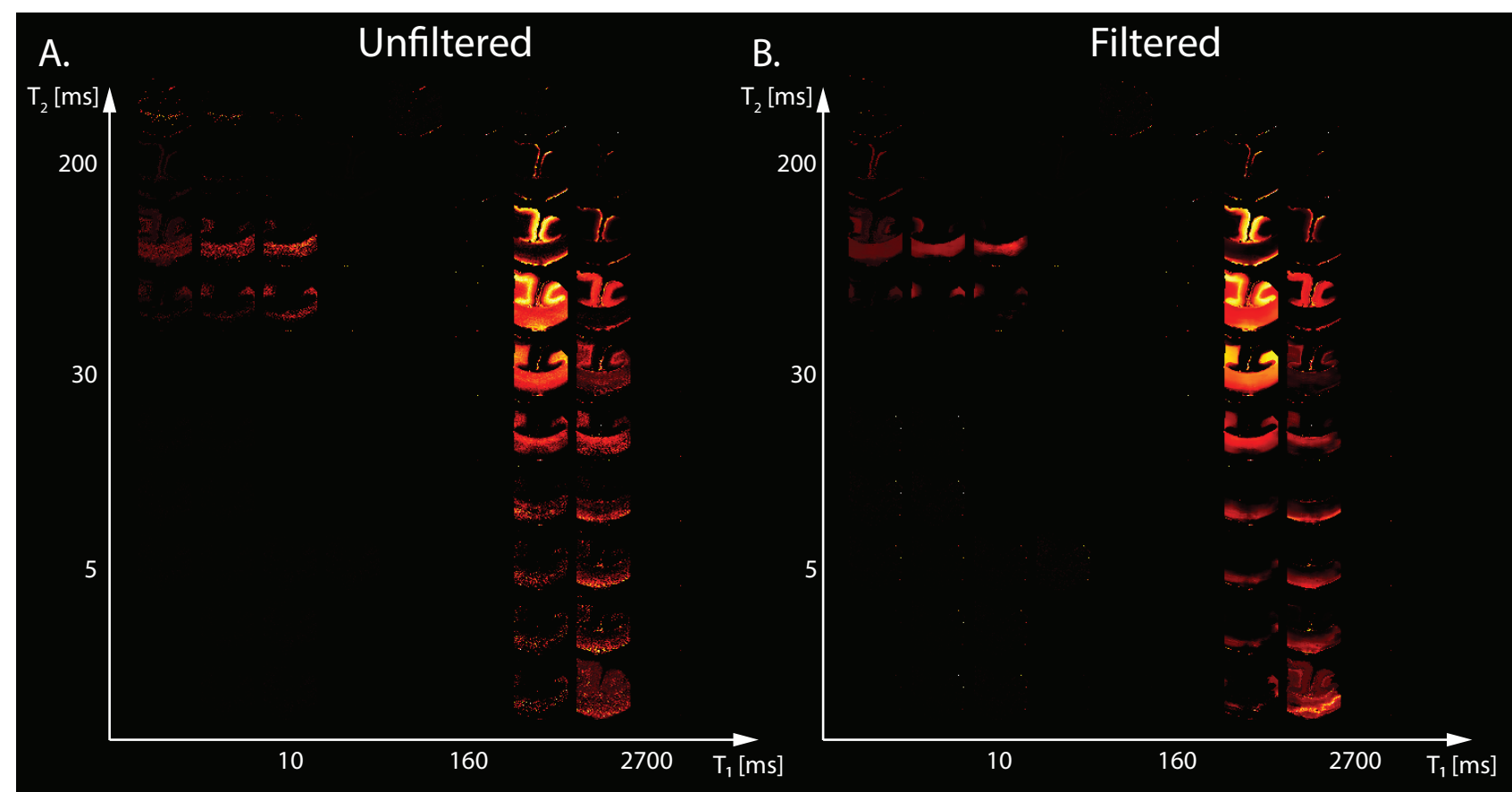

C.

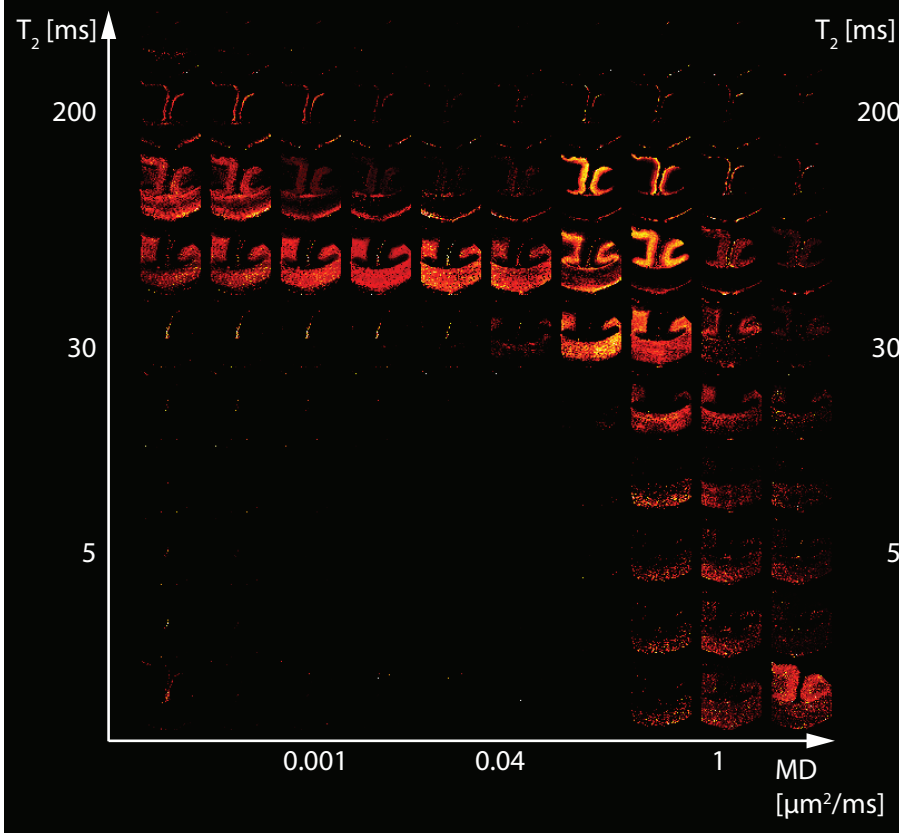

D.

$[\mathrm{ms}] \Lambda$

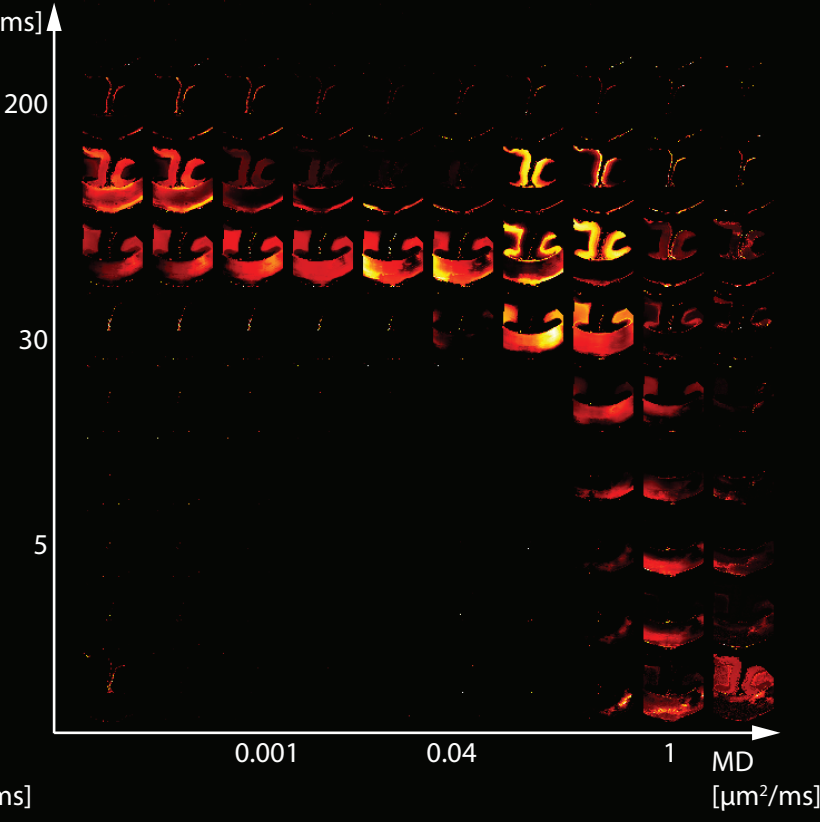

Figure 1. Maps of 2D probability density functions (i.e., 2D normalized spectra) from Subject 1 (control) of (A) unfiltered and (B) filtered subvoxel $T_{1}-T_{2}$ values reconstructed on a $10 \times 10$ grid of subvoxel $T_{1}$ values (horizontal axes) and subvoxel $T_{2}$ values (vertical axes), and maps of (C) unfiltered and (D) filtered subvoxel MD- $T_{2}$ values reconstructed on a $10 \times 10$ grid of subvoxel MD values (horizontal axes) and subvoxel $T_{2}$ values (vertical axes).

The spatially-resolved subvoxel $T_{1}-T_{2}$ and MD- $T_{2}$ spectral components from the first TBI case (Subject 2) are shown in Fig. 2. As before, the left column shows the results from the unfiltered data $\left(T_{1}-T_{2}\right.$ and 174 MD $-T_{2}$ in Figs. 2A and C, respectively), and the right column shows the results from the filtered data 175 ( $T_{1}-T_{2}$ and MD- $T_{2}$ in Figs. $2 \mathrm{~B}$ and $\mathrm{D}$, respectively). Similarly to the control case, here too the maps 176 revealed signal components that were spatially consistent with specific tissue types. 


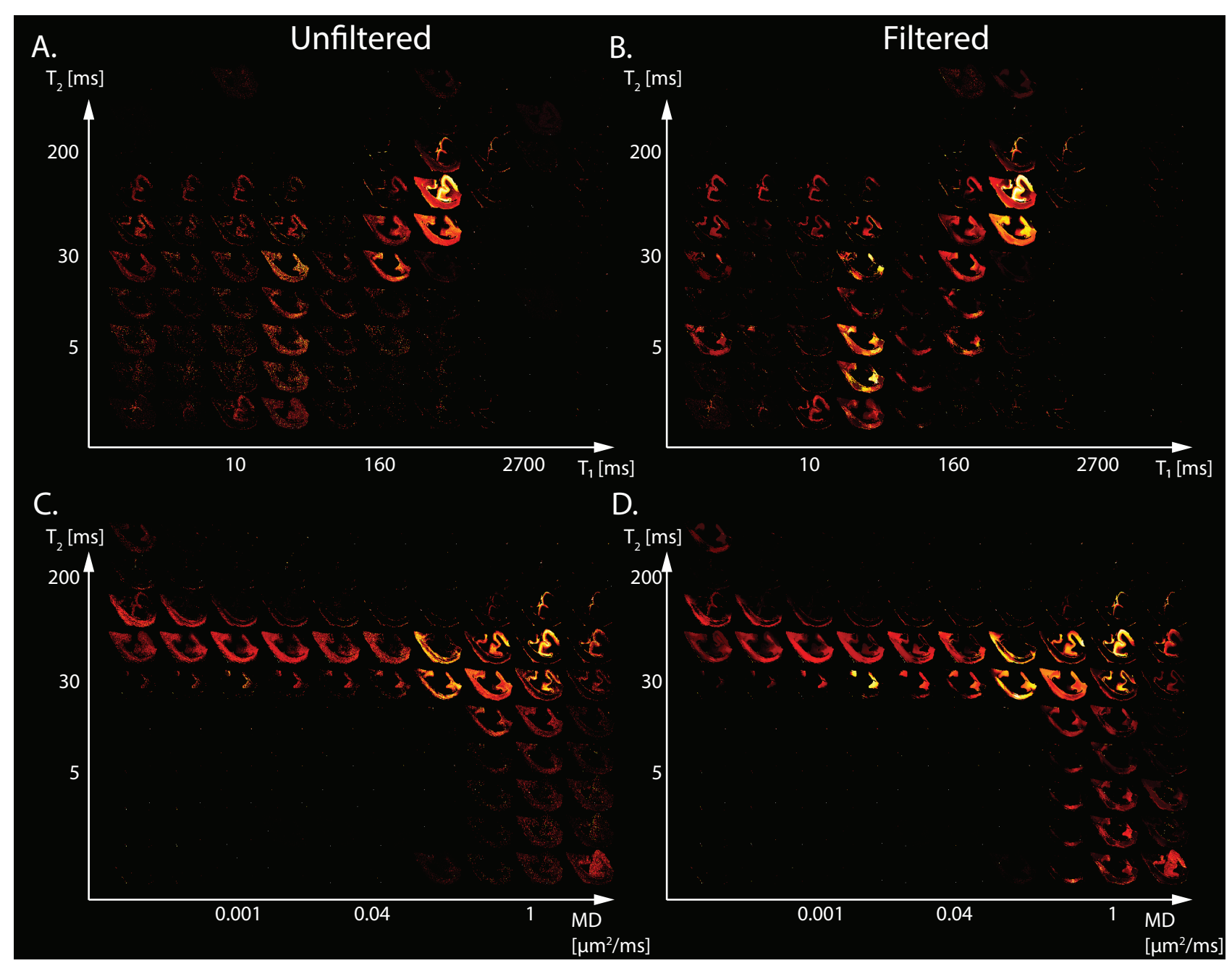

Figure 2. Maps of 2D probability density functions (i.e., 2D normalized spectra) from Subject 2 (TBI) of (A) unfiltered and (B) filtered subvoxel $T_{1}-T_{2}$ values reconstructed on a $10 \times 10$ grid of subvoxel $T_{1}$ values (horizontal axes) and subvoxel $T_{2}$ values (vertical axes), and maps of (C) unfiltered and (D) filtered subvoxel MD- $T_{2}$ values reconstructed on a $10 \times 10$ grid of subvoxel MD values (horizontal axes) and subvoxel $T_{2}$ values (vertical axes).

The spatially-resolved subvoxel $T_{1}-T_{2}$ and MD- $T_{2}$ spectral components from the second TBI case (Subject 3) are shown in Fig. 3. Unfiltered ( $T_{1}-T_{2}$ and MD- $T_{2}$ in Figs. 3 A and C, respectively) and filtered data $\left(T_{1}-T_{2}\right.$ and MD- $T_{2}$ in Figs. $3 \mathrm{~B}$ and $\mathrm{D}$, respectively) are shown. As before, signal components that were spatially consistent with specific tissue types as a function of $T_{1}, T_{2}$, and MD were revealed.

Figure 4 shows histological images and multidimensional MR-derived injury biomarker maps of the three representative cases. Histological images (red = APP stain) of the control case (Subject 1) show negative APP staining, compared with positive APP staining in the injured samples (Subjects 2 and 3). We then examine separately the two MRI-derived injury biomarkers, $T_{1}-T_{2}$ and MD- $T_{2}$, and show the resulting images obtained using the unfiltered full dataset (as originally published in [22]), the filtered full dataset, and the filtered reduced dataset. In addition, the MRI-derived injury biomarkers obtained by using the unfiltered reduced dataset are shown in Fig. S1 in the Supplementary Material.

Visual inspection of the different injury biomarker maps shown in Figs. 4 and S1 revealed that filtering of the data does not result in loss of the spectral information of interest, and furthermore, the filtered images 


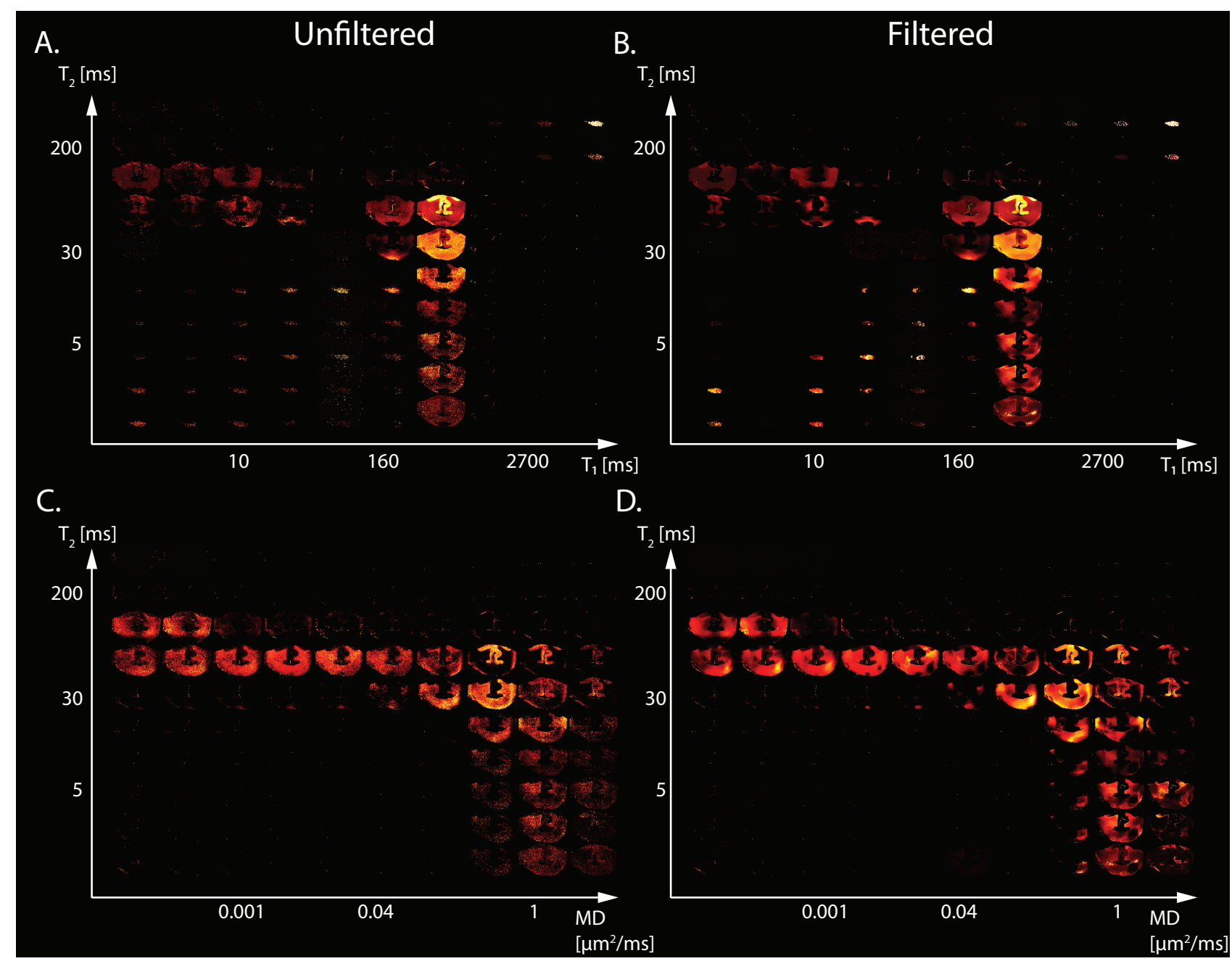

Figure 3. Maps of 2D probability density functions (i.e., 2D normalized spectra) from Subject 3 (TBI) of (A) unfiltered and (B) filtered subvoxel $T_{1}-T_{2}$ values reconstructed on a $10 \times 10$ grid of subvoxel $T_{1}$ values (horizontal axes) and subvoxel $T_{2}$ values (vertical axes), and maps of (C) unfiltered and (D) filtered subvoxel MD- $T_{2}$ values reconstructed on a $10 \times 10$ grid of subvoxel MD values (horizontal axes) and subvoxel $T_{2}$ values (vertical axes).

appear qualitatively of higher quality. Importantly, the data reduction in the case of the filtered data did not significantly affect the resulting injury biomarker maps (Fig. 4).

\subsection{Evaluation of performance and correlation with histology}

Evaluation of filtering performance was based upon the extent of noise reduction and feature preservation, and was quantified by computing the structural similarity index (SSIM) values [33] between the injury biomarker maps under the different experimental conditions (e.g., unfiltered, filtered) and the co-registered APP density histological image as reference. All of the SSIM values are shown in Fig. 5. In the context of the current study we are most interested in the ability to accelerate the multidimensional MRI acquisition, and therefore the accuracy and quality of the reduced data cases are of particular importance. Compared with the unfiltered and reduced data injury biomarker maps, the SSIM values of the filtered and reduced data images increased by $11.1 \%, 0.9 \%$, and $14.3 \%$ for the MD- $T_{2}$-based biomarker for Subjects 1 to 3 , respectively, and increased by $8.6 \%, 7.7 \%$, and $4.6 \%$ for the $T_{1}-T_{2}$-based biomarker for Subjects 1 to 3 , respectively. All of these increases in SSIM were statistically significant $(p<0.001)$. 
bioRxiv preprint doi: https://doi.org/10.1101/2021.07.06.451291; this version posted August $13,2021$. The copyright holder for this preprint (which was not certified by peer review) is the author/funder. This article is a US Government work. It is not subject to copyright under 17 USC 105 and is also made available for use under a CCO license.

Benjamini et al.

Accelerated multidimensional MRI

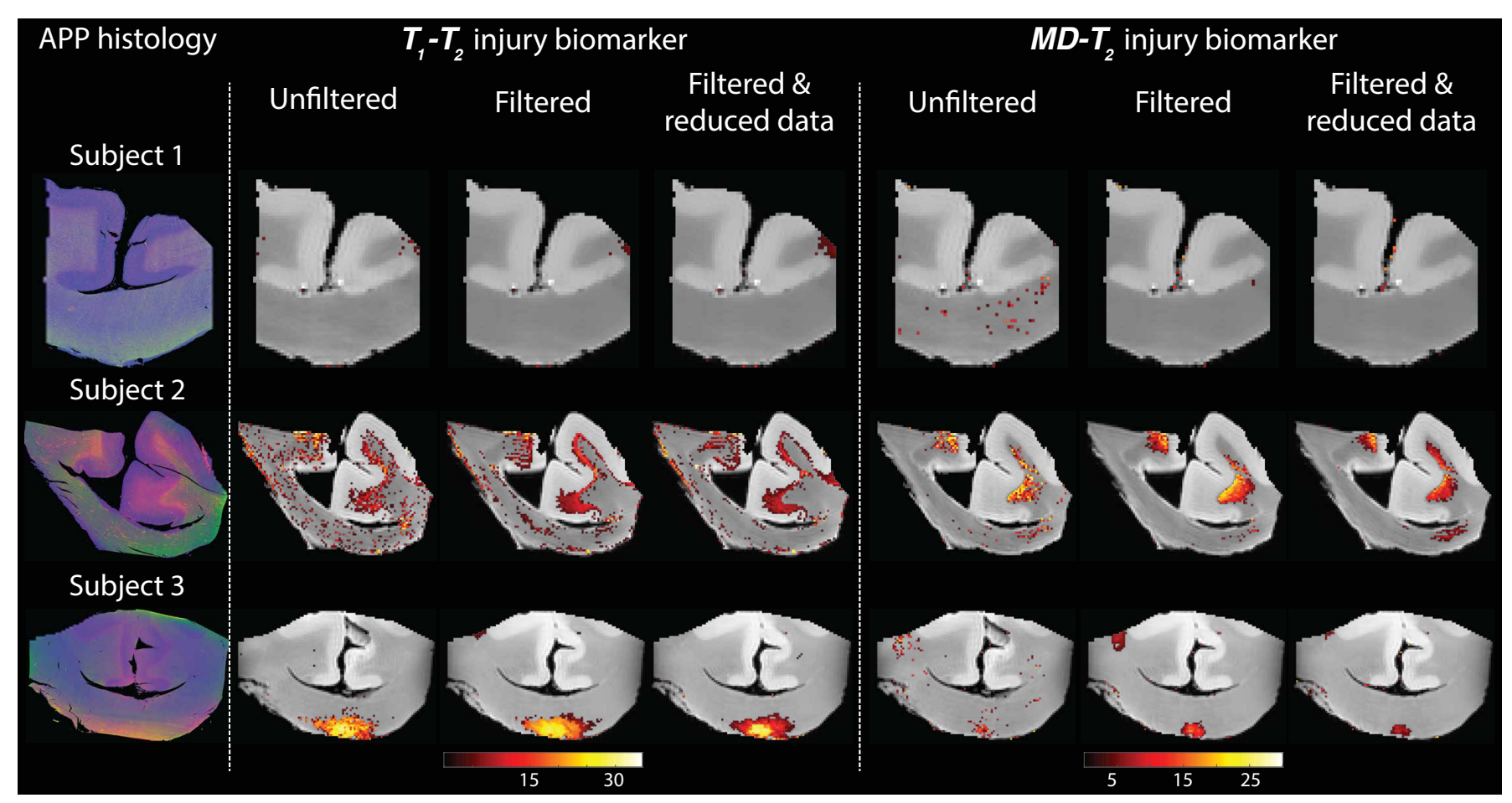

Figure 4. Histological images and multidimensional MR-derived injury biomarker maps of three representative cases, and under different conditions (left to right: unfiltered, filtered, and filtered \& reduced data). Deconvolved histological APP images (co-registered with the MRI) are shown on the left panel, red = APP stain (top to bottom: control, and two TBI cases). All multidimensional injury maps were thresholded at $10 \%$ of the maximal intensity and overlaid on grayscale proton density images. Multidimensional injury maps of Subject 1 (control) show absent of significant injury under all experimental conditions. Multidimensional injury maps of Subject 2 (TBI) show substantial injury along the white-gray matter interface under all experimental conditions. Multidimensional injury maps of Subject 3 (TBI) show substantial injury at the bottom of the CC under all experimental conditions.

To further evaluate the performance of the NESMA filter and the subsequent data reduction, we performed radiological-pathological correlation analyses with histological APP density and all the investigated MRI parameters under the different experimental conditions (e.g., MD- $T_{2}$ unfiltered, $T_{1}-T_{2}$ filtered \& reduced). Figure 6 summarizes the association between the investigated MR metrics and the pathological findings in normal WM, cortical GM, and TAI ROIs.

To assess the relationship of the MRI parameters with the degree of injury, all tissue ROIs were grouped together and correlated with the APP density (solid lines in Fig. 6). All multidimensional injury biomarker cases under every experimental conditions were strongly and significantly positively correlated with the APP density (i.e., \% area APP). These correlations illustrate how the multidimensional injury biomarker maps provide "true negative", in the sense that any region outside of the TAI lesions has zero or close to zero intensity.

Of particular interest was the case of the MD- $T_{2}$ injury biomarker, where the unfiltered dataset resulted 215 in relatively scattered correlation $\left(r=0.565, p<2 \times 10^{-4}\right.$, Fig. 6A), which was largely unaffected by 216 the data reduction $\left(r=0.557, p<3 \times 10^{-4}\right.$, Fig. 6B). Marked improvement was observed after filtering 217 the data, which led to significantly tighter correlation between the MRI biomarker and the histological 218 marker $\left(r=0.812, p<2 \times 10^{-9}\right.$, Fig. $6 \mathrm{C}$ ). As can be expected, the $59.6 \%$ reduction in the data amount 219 led to some reduction in the radiological-pathological correlation $\left(r=0.723, p<4 \times 10^{-7}\right.$, Fig. 6D), 


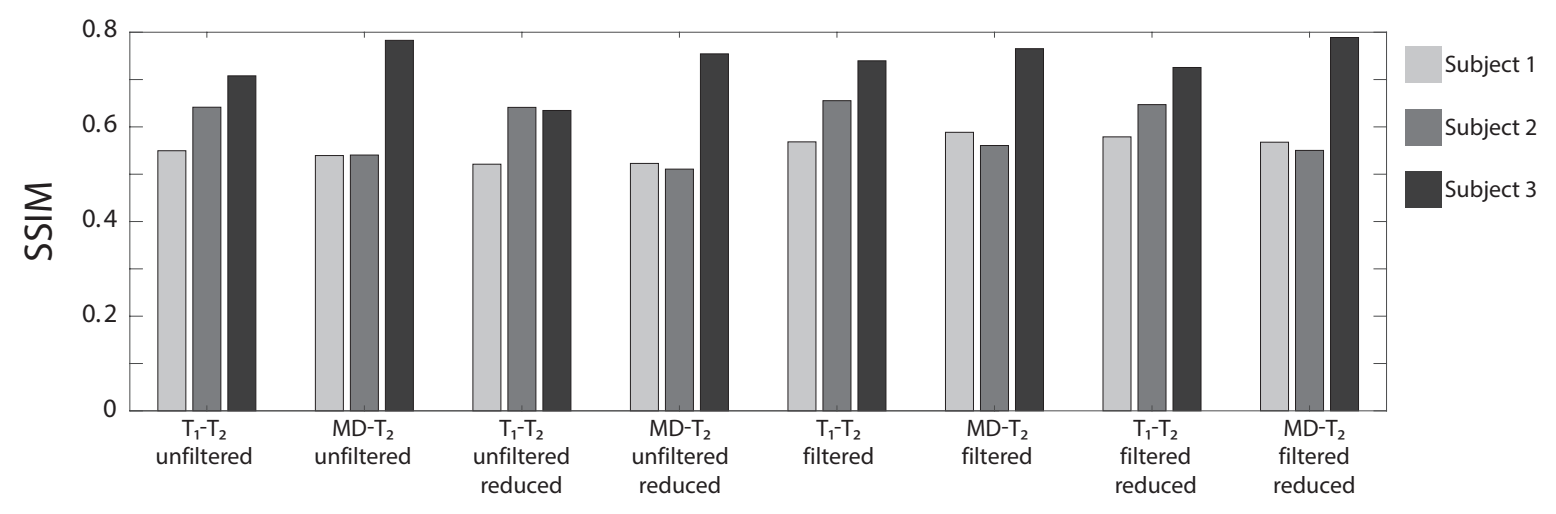

Figure 5. The structural similarity index (SSIM) values between the injury biomarker images under the different experimental conditions (e.g., $T_{1}-T_{2}$ unfiltered, MD- $T_{2}$ filtered reduced data) and the co-registered APP density histological image as reference. The three bars at each condition represent the different Subjects $($ blue $=$ Subject 1 , red $=$ Subject 2, and yellow $=$ Subject 3 ).

although still performing better than the unfiltered full MD- $T_{2}$ dataset. Furthermore, filtering led to a reduced variance within the ROIs, as seen by the decrease in the 95\% confidence intervals (error bars in Fig. 6).
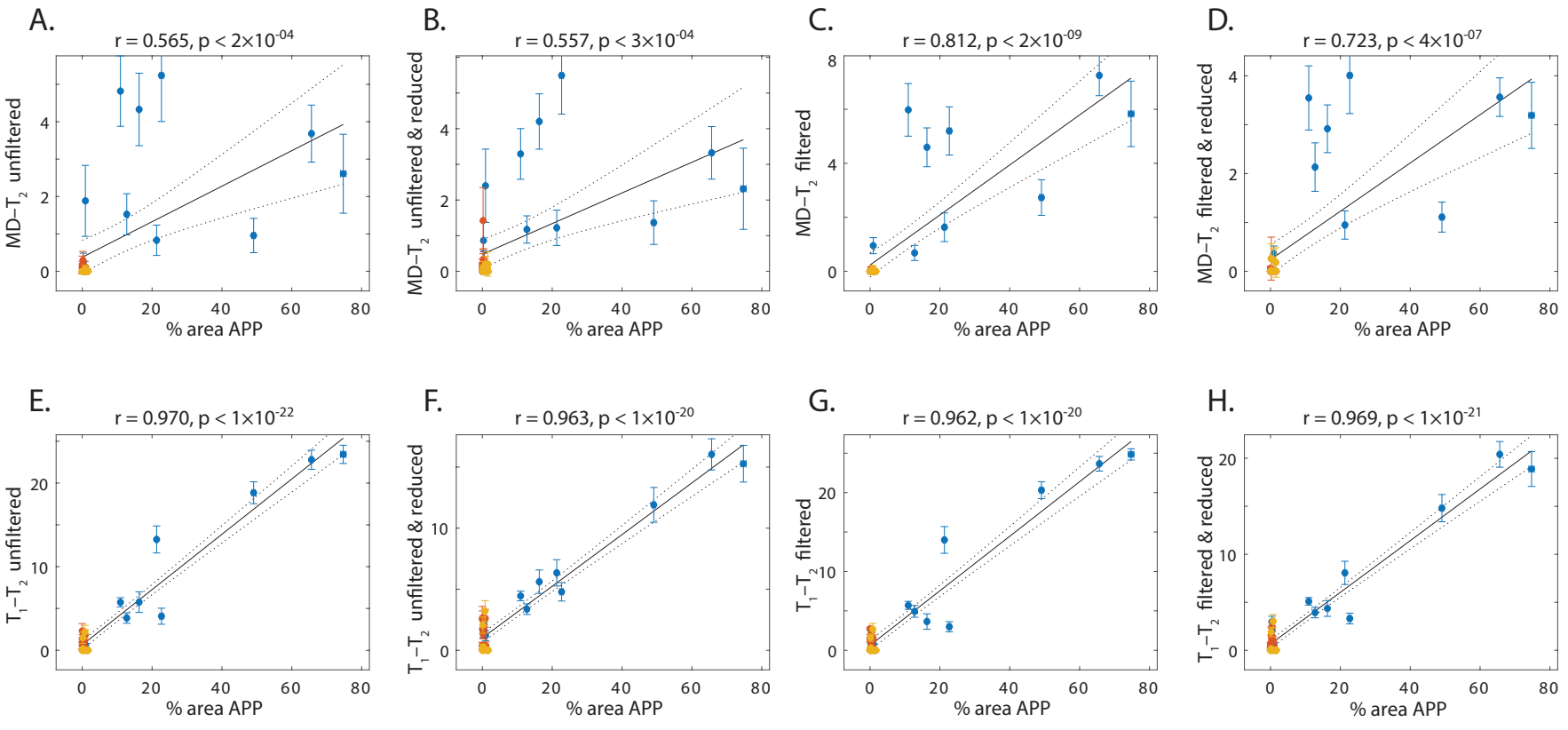

- Traumatic axonal injury (TAI)

White matter (WM)

- Gray matter (GM)

linear fit (TAl+WM+GM)

Figure 6. APP density (\% area) from 36 tissue regions (APP-positive regions from each TBI case, WM and GM regions), and its correlation with injury biomarker parameter under different experimental conditions. Individual data points represent the mean ROI value from each post-mortem tissue sample. Scatterplots of the mean (with 95\% confidence interval error bars) $\%$ area APP and (A) MD- $T_{2}$ unfiltered, (B) MD- $T_{2}$ unfiltered \& reduced, (C) MD- $T_{2}$ filtered, (D) MD- $T_{2}$ filtered \& reduced, (E) $T_{1}-T_{2}$ unfiltered, (F) $T_{1}-T_{2}$ unfiltered $\&$ reduced, (G) $T_{1}-T_{2}$ filtered, and (H) $T_{1}-T_{2}$ filtered $\&$ reduced, show positive and significant correlation with APP density. 
The $T_{1}-T_{2}$ injury biomarker derived from the unfiltered dataset had excellent correlation with histological APP density $\left(r=0.970, p<1 \times 10^{-22}\right.$, Fig. $\left.6 \mathrm{E}\right)$, and therefore, it is not surprising that this strong relationship was maintained under all of the investigated experimental conditions $\left(r=0.963, p<1 \times 10^{-20}\right.$, $r=0.962, p<1 \times 10^{-20}$, and $r=0.969, p<1 \times 10^{-21}$, Figs. $6 \mathrm{~F}-\mathrm{H}$, for unfiltered $\&$ reduced, filtered, and filtered $\&$ reduced, respectively).

\section{DISCUSSION}

Here we report the use of the NESMA filter on multidimensional MRI data, in particular voxelwise $T_{1}-T_{2}$ and MD- $T_{2}$ spectra in fixed human Corpus Callosum, to remove noise and reduce total scan time. We focused on results from a recent study that identified potential imaging biomarkers of axonal injury pathology from the joint analysis of multidimensional MRI and histopathological data [22]. These axonal injury maps were shown to be significantly and strongly correlated with histological evidence of axonal injury. Reprocessing these data provided an opportunity to test the performance of the NESMA filter and its effect on the accuracy of the injury biomarker maps, relative to the histological reference.

Our findings showed that noise reduction in the multidimensional MRI data using an adaptive nonlocal multispectral filter (i.e., NESMA [20]) improved the accuracy of the resulting injury biomarker maps, and furthermore, allowed for data reduction of $35.7 \%$ and $59.6 \%$ from the full dataset, which led to using only 36 and 122 images in the $T_{1}-T_{2}$ and MD- $T_{2}$ cases, respectively.

Specifically, visual inspection and a side-by-side comparison of the unfiltered and filtered subvoxel $T_{1}-T_{2}$ and MD- $T_{2}$ spectral components (Figs. 1, 2, and 3) showed that the filtered maps exhibit lower random variations, in particular at the lower ends of the spectra, and that there was no apparent loss of spectral information. For example, Subject 3 exhibited a relatively focal axonal injury at the bottom of the CC (Fig. 4. left panel), captured at the lower end of the $T_{1}-T_{2}$ spectra, which was previously associated with axonal injury [22]. Noticeable noise reduction at these spectral lower ends was observed, which is crucial to the robust identification of axonal injury from these multidimensional MRI data.

Visual inspection of the resulting $T_{1}-T_{2}$ and MD- $T_{2}$ injury biomarker maps with respect to co-registered APP histological images suggested improved accuracy after applying the NESMA filter, even after the data was reduced (Figs. 4). Quantitative evaluation that compared the SSIM between the co-registered APP histological images and injury biomarker maps derived from unfiltered and filtered reduced datasets showed a significant increase as a result of the filtering across all subjects (Fig. 5).

We performed radiological-pathological correlation analyses with histological APP density and all the investigated MRI parameters under the different experimental conditions to assess quantitatively whether and to what extent the proposed approach preserves strong correlations even under substantial data reduction (Fig. 6). This analysis indicated that not only the correlations were preserved, but furthermore, they were considerably improved, even after data reduction, as a result of filtering the data. Lastly, our results suggest that the previously proposed [22] adaptive method of locating the injury-associated $T_{1}-T_{2}$-MD spectral signature is robust to noise removal procedures and to data reduction.

Common to all ex vivo human MRI studies, our data include the effects of post-mortem degeneration, fixation and resulting dehydration. Because $T_{1}, T_{2}$, and diffusion dynamics are different in fixed tissue compared with living systems, further investigation will be needed to establish whether and how the axonal injury-related $T_{1}-T_{2}-\mathrm{MD}$ range of multidimensional magnetic resonance parameters is altered in vivo. In this context it is important to note that our findings are not based on absolute values of $T_{1}, T_{2}$, and MD, which indeed are expected to change in vivo. Instead, all of the injury biomarkers maps are generated using 
264 the relative signal fraction of an automatically identified $T_{1}-T_{2}$-MD range that does not depend on the

\section{AUTHOR CONTRIBUTIONS}

\section{FUNDING} diagnostic multidimensional MRI protocols.

The performance of the NESMA filter does not depend on the particular multidimensional spectra quantification processing pipeline because the filter is applied in the image domain, before its transformed into voxelwise spectra. Here we applied a constrained $\ell_{2}$ regularized inversion framework [13], however, we anticipate that the demonstrated improvement after filtering could be extended to other approaches such as $\ell_{1}$ regularization [34], Monte-Carlo inversion [35, 36], and InSpect [16].

Multidimensional MRI is an emerging approach [37] that is now being applied to address a range of medical conditions such as prediction of pregnancy complications via placenta characterization [9], spinal cord injury [6, 38], prostate cancer [39], breast cancer [40], and axonal injury due to TBI [22]. Recent in vivo proof-of-concept applications of subvoxel $T_{1}-T_{2}$ correlation spectra using 105 images [41] and of subvoxel diffusion- $T_{1}$ correlation spectra using 363 [11] and 304 [42] images are promising. Here we showed that accurate and robust subvoxel $T_{1}-T_{2}$ and $\mathrm{MD}-T_{2}$ correlation spectra can be obtained using only 36 and 122 images, respectively, by using a constrained optimization data processing framework (i.e., MADCO [13]) in conjunction with applying the NESMA filter to reduce noise. A reliable and robust noise removal and consequent acquisition acceleration should further advance the field towards clinically-feasible

\section{CONFLICT OF INTEREST STATEMENT}

The authors declare that the research was conducted in the absence of any commercial or financial relationships that could be construed as a potential conflict of interest.

DB: conceptualization, design of the study, methodology, software, investigation, data curation, writing - original draft, writing — review and editing, visualization, supervision, and project administration. MB: conceptualization, design of the study, methodology, software, and writing - review and editing. MK: methodology, investigation, and writing — review and editing. DI: methodology, investigation, and writing — review and editing. DP: investigation, methodology, resources, and writing — review and editing. DLB: design of the study, investigation, resources, and writing - review and editing. PB: conceptualization, methodology, resources, writing - review and editing, and funding acquisition. All authors contributed to the article and approved the submitted version.

This research was partially supported by a grant from the U.S. Department of Defense, Program Project 308430 Uniformed Services University of the Health Sciences (USUHS). Support for this work also included funding from the U.S. Department of Defense to the Brain Tissue Repository and Neuropathology Program, Center for Neuroscience and Regenerative Medicine (CNRM). DB and MEK were supported by the CNRM Neuroradiology- Neuropathology Correlations Core. MB was supported by the Intramural Research Program of the National Institute on Aging. DI, DPP, and DLB were supported by the CNRM and USUHS. PJB was supported by the Intramural Research Program of the Eunice Kennedy Shriver National Institute of Child Health and Human Development. 


\section{ACKNOWLEDGMENTS}

We thank the subjects' families that consented for brain donations for the better understanding of TBI consequences. The authors thank Mrs Patricia Lee, Mrs Nichelle Gray and Mr Paul Gegbeh for their valuable technical work. We are grateful to Mrs Stacey Gentile, Mrs Deona Cooper and $\mathrm{Mr}$ Harold Kramer Anderson for their administrative assistance. We thank the TRACK-TBI Investigators (https://tracktbi.ucsf.edu/transforming-research-and-clinical-knowledge-tbi).

The opinions expressed herein are those of the authors and are not necessarily representative of those of the Uniformed Services University of the Health Sciences (USUHS), the Department of Defense (DOD), the NIH or any other US government agency.

\section{SUPPLEMENTAL DATA}

Supplementary material is available online.

\section{DATA AVAILABILITY STATEMENT}

The datasets generated and analyzed during the current study are available from the corresponding author on reasonable request.

\section{REFERENCES}

[1] English AE, Whittall KP, Joy MLG, Henkelman RM. Quantitative Two-Dimensional time Correlation Relaxometry. Magnetic Resonance in Medicine 22 (1991) 425-434.

[2] Hürlimann M, Flaum M, Venkataramanan L, Flaum C, Freedman R, Hirasaki G. Diffusion-relaxation distribution functions of sedimentary rocks in different saturation states. Magnetic Resonance Imaging 21 (2003) 305-310. doi:10.1016/S0730-725X(03)00159-0.

[3] Topgaard D. Multidimensional diffusion MRI. Journal of Magnetic Resonance 275 (2017) 98-113. doi:10.1016/j.jmr.2016.12.007.

[4] Benjamini D, Basser PJ. Multidimensional correlation MRI. NMR in Biomedicine (2020) e4226. doi:10.1002/nbm.4226.

[5] de Almeida Martins JP, Topgaard D. Two-Dimensional Correlation of Isotropic and Directional Diffusion Using NMR. Physical Review Letters 116 (2016) 087601.

[6] Kim D, Doyle EK, Wisnowski JL, Kim JH, Haldar JP. Diffusion-relaxation correlation spectroscopic imaging: A multidimensional approach for probing microstructure. Magnetic Resonance in Medicine 78 (2017) 2236-2249. doi:10.1002/mrm.26629.

[7] Benjamini D, Basser P. Water mobility spectral imaging of the spinal cord: Parametrization of model-free Laplace MRI. Magnetic Resonance Imaging 56 (2019).

[8] Benjamini D, Basser PJ. Magnetic resonance microdynamic imaging reveals distinct tissue microenvironments. NeuroImage 163 (2017) 183-196. doi:10.1016/j.neuroimage.2017.09.033.

[9] Slator PJ, Hutter J, Palombo M, Jackson LH, Ho A, Panagiotaki E, et al. Combined diffusionrelaxometry MRI to identify dysfunction in the human placenta. Magnetic Resonance in Medicine $\mathbf{8 2}$ (2019) 95-106. doi:10.1002/mrm.27733.

[10] de Almeida Martins JP, Tax CMW, Reymbaut A, Szczepankiewicz F, Chamberland M, Jones DK, et al. Computing and visualising intra-voxel orientation-specific relaxation-diffusion features in the human brain. Human Brain Mapping 42 (2021) 310-328.

[11] Reymbaut A, Critchley J, Durighel G, Sprenger T, Sughrue M, Bryskhe K, et al. Toward nonparametric diffusion- characterization of crossing fibers in the human brain. Magnetic Resonance in Medicine $\mathbf{8 5}$ (2021) 2815-2827. 
[12] Benjamini D. Nonparametric Inversion of Relaxation and Diffusion Correlation Data. Topgaard D, editor, Advanced Diffusion Encoding Methods in MRI (Cambridge, UK: Royal Society of Chemistry), chap. 10 (2020), 278-316.

[13] Benjamini D, Basser P. Use of marginal distributions constrained optimization (MADCO) for accelerated 2D MRI relaxometry and diffusometry. Journal of Magnetic Resonance 271 (2016) 40-45. doi:10.1016/j.jmr.2016.08.004.

[14] Benjamini D, Basser PJ. Towards clinically feasible relaxation-diffusion correlation MRI using MADCO. Microporous and Mesoporous Materials 269 (2018) 93-96. doi:10.1016/j.micromeso.2017. 02.001 .

[15] Bai R, Cloninger A, Czaja W, Basser PJ. Efficient 2D MRI relaxometry using compressed sensing. Journal of Magnetic Resonance 255 (2015) 88-99.

[16] Slator PJ, Hutter J, Marinescu RV, Palombo M, Jackson LH, Ho A, et al. Data-Driven multiContrast spectral microstructure imaging with InSpect: INtegrated SPECTral component estimation and mapping. Medical Image Analysis 71 (2021) 102045.

[17] Hutter J, Slator PJ, Christiaens D, Teixeira RPAG, Roberts T, Jackson L, et al. Integrated and efficient diffusion-relaxometry using ZEBRA. Scientific Reports 8 (2018) 15138. doi:10.1038/ s41598-018-33463-2.

[18] Manhard MK, Stockmann J, Liao C, Park D, Han S, Fair M, et al. A multi-inversion multi-echo spin and gradient echo echo planar imaging sequence with low image distortion for rapid quantitative parameter mapping and synthetic image contrasts. Magnetic Resonance in Medicine 86 (2021) 866-880.

[19] Bouhrara M, Bonny JM, Ashinsky BG, Maring MC, Spencer RG. Noise Estimation and Reduction in Magnetic Resonance Imaging Using a New Multispectral Nonlocal Maximum-likelihood Filter. IEEE Transactions on Medical Imaging 36 (2017) 181-193.

[20] Bouhrara M, Reiter DA, Maring MC, Bonny JM, Spencer RG. Use of the NESMA Filter to Improve Myelin Water Fraction Mapping with Brain MRI. Journal of Neuroimaging 28 (2018) 640-649.

[21] Bouhrara M, Lee DY, Rejimon AC, Bergeron CM, Spencer RG. Spatially adaptive unsupervised multispectral nonlocal filtering for improved cerebral blood flow mapping using arterial spin labeling magnetic resonance imaging. Journal of Neuroscience Methods 309 (2018) 121-131.

[22] Benjamini D, Iacono D, Komlosh ME, Perl DP, Brody DL, Basser PJ. Diffuse axonal injury has a characteristic multidimensional MRI signature in the human brain. Brain 144 (2021) 800-816.

[23] Johnson VE, Stewart W, Smith DH. Axonal pathology in traumatic brain injury. Experimental Neurology 246 (2013) 35-43. doi:10.1016/j.expneurol.2012.01.013.

[24] Pas K, Komlosh ME, Perl DP, Basser PJ, Benjamini D. Retaining information from multidimensional correlation MRI using a spectral regions of interest generator. Scientific Reports 10 (2020) 3246. doi:10.1038/s41598-020-60092-5.

[25] Provencher SW. A constrained regularization method for inverting data represented by linear algebraic or integral equations. Computer Physics Communications 27 (1982) 213-227. doi:10. 1016/0010-4655(82)90173-4.

[26] Kroeker RM, Henkelman MR. Analysis of biological NMR relaxation data with continuous distributions of relaxation times. Journal of Magnetic Resonance (1969) 69 (1986) 218-235. doi:10.1016/0022-2364(86)90074-0.

[27] Song YQ, Venkataramanan L, Hürlimann M, Flaum M, Frulla P, Straley C. T1-T2 Correlation Spectra Obtained Using a Fast Two-Dimensional Laplace Inversion. Journal of Magnetic Resonance 154 (2002) 261-268. doi:10.1006/jmre.2001.2474. 
[28] Mitchell J, Chandrasekera TC, Gladden LF. Numerical estimation of relaxation and diffusion distributions in two dimensions. Progress in Nuclear Magnetic Resonance Spectroscopy 64 (2012) 34-50. doi:10.1016/j.pnmrs.2011.07.002.

[29] Celik H, Bouhrara M, Reiter DA, Fishbein KW, Spencer RG. Stabilization of the inverse Laplace transform of multiexponential decay through introduction of a second dimension. Journal of Magnetic Resonance 236 (2013) 134-139. doi:10.1016/j.jmr.2013.07.008.

[30] Labadie C, Lee J, Vetek G, Springer C. Relaxographic Imaging. Journal of Magnetic Resonance, Series B 105 (1994) 99-112. doi:10.1006/jmrb.1994.1109.

[31] Macenko M, Niethammer M, Marron JS, Borland D, Woosley JT, Xiaojun Guan, et al. A method for normalizing histology slides for quantitative analysis. 2009 IEEE International Symposium on Biomedical Imaging: From Nano to Macro (IEEE) (2009), 1107-1110. doi:10.1109/ISBI.2009. 5193250.

[32] Ruifrok AC, Johnston DA. Quantification of histochemical staining by color deconvolution. Analytical and Quantitative Cytology and Histology 23 (2001) 291-299.

[33] Wang Z, Bovik A, Sheikh H, Simoncelli E. Image Quality Assessment: From Error Visibility to Structural Similarity. IEEE Transactions on Image Processing 13 (2004) 600-612.

[34] Reci A, Sederman A, Gladden L. Obtaining sparse distributions in 2D inverse problems. Journal of Magnetic Resonance 281 (2017) 188-198.

[35] Prange M, Song YQ. Quantifying uncertainty in NMR T2 spectra using Monte Carlo inversion. Journal of Magnetic Resonance 196 (2009) 54-60.

[36] de Almeida Martins JP, Topgaard D. Multidimensional correlation of nuclear relaxation rates and diffusion tensors for model-free investigations of heterogeneous anisotropic porous materials. Scientific Reports 8 (2018) 2488.

[37] Slator PJ, Palombo M, Miller KL, Westin C, Laun F, Kim D, et al. Combined diffusion-relaxometry microstructure imaging: Current status and future prospects. Magnetic Resonance in Medicine (2021). doi:10.1002/mrm.28963.

[38] Benjamini D, Hutchinson EB, Komlosh ME, Comrie CJ, Schwerin SC, Zhang G, et al. Direct and specific assessment of axonal injury and spinal cord microenvironments using diffusion correlation imaging. NeuroImage 221 (2020) 117195.

[39] Zhang Z, Wu HH, Priester A, Magyar C, Afshari Mirak S, Shakeri S, et al. Prostate Microstructure in Prostate Cancer Using 3-T MRI with Diffusion-Relaxation Correlation Spectrum Imaging: Validation with Whole-Mount Digital Histopathology. Radiology 296 (2020) 348-355.

[40] Naranjo ID, Reymbaut A, Brynolfsson P, Lo Gullo R, Bryskhe K, Topgaard D, et al. Multidimensional Diffusion Magnetic Resonance Imaging for Characterization of Tissue Microstructure in Breast Cancer Patients: A Prospective Pilot Study. Cancers 13 (2021) 1606.

[41] Kim D, Wisnowski JL, Nguyen CT, Haldar JP. Multidimensional correlation spectroscopic imaging of exponential decays: From theoretical principles to in vivo human applications. NMR in Biomedicine e4244 (2020). doi:10.1002/nbm.4244.

[42] Avram AV, Sarlls JE, Basser PJ. Whole-Brain Imaging of Subvoxel T1-Diffusion Correlation Spectra in Human Subjects. Frontiers in Neuroscience 15 (2021). 